\title{
Second generation degrader foil for the CARIBU project
}

\author{
Matthew Gott*, John Greene, Brad DiGiovine, Guy Savard, and Bruce Zabransky \\ Physics Division, Argonne National Laboratory, Lemont, Illinois, United States
}

\begin{abstract}
The Californium Rare Ion Breeder Upgrade (CARIBU) project utilizes ${ }^{252} \mathrm{Cf}$ to provide beams of neutron-rich nuclides with intensities not currently available at other facilities. The electroplated ${ }^{252} \mathrm{Cf}$ source is positioned inside a large helium gas catcher, where the ejected fission fragments lose their energy and are slowed. Prior to entering this gas catcher, the ions first pass through a gold cover foil to contain self-sputtering recoil emissions and then through an aluminium degrader foil, where much of their residual energy is reduced. In the past due to production limitations, a less than ideal cylindrical shaped degrader was utilized. This resulted in non-uniform energy loss as the ions passed through the degrader. With the advent of $3 \mathrm{D}$ printing, a new hemispherical degrader was prepared to enable a more uniform energy loss. The design, production, and assembly will be discussed.
\end{abstract}

\section{Introduction and motivation}

Beams of exotic neutron-rich nuclei are of high interest for nuclear physics research studying phenomena such as nucleosynthesis of heavy nuclei and nuclear structure of nuclides approaching the neutron dripline. The Californium Rare Ion Breeder Upgrade (CARIBU) project utilizes ${ }^{252} \mathrm{Cf}$ to provide radioactive beams not produced in the low-energy fission of uranium as well as producing elements that are difficult to extract using standard ISOL techniques [1]. CARIBU provides beams of neutron-rich species to the Argonne Tandem Linear Accelerator System (ATLAS) where they can be accelerated up to $\sim 10 \mathrm{MeV} / u$. The ${ }^{252} \mathrm{Cf}$ source is positioned inside a large RF gas catcher filled with highpurity helium, where the fission fragments lose energy and are slowed down. Once extracted, the ions are accelerated to the isobar separator, where two $60^{\circ}$ magnets are used to isolate the species of interest; this high-purity beam is then sent directly or reaccelerated using the EBIS charge breeder to various experimental stations at ATLAS.

\subsection{The ${ }^{252} \mathrm{Cf}$ source}

The ${ }^{252} \mathrm{Cf}$ source $\left(\mathrm{t}_{1 / 2}=2.645 \mathrm{a} ; 3.1 \%\right.$ fission branch) used in this system was prepared and provided by Oak Ridge National Laboratory. The ${ }^{252} \mathrm{Cf}$ is produced by successive neutron capture on an actinide target (originally primarily plutonium) using the High Flux Isotope Reactor, one of the highest flux reactors in the world [2]. The material is electroplated onto a stainless steel plate providing a fission fragment source for CARIBU. The current source is $500 \mathrm{mCi}$ in total activity but effectively $130 \mathrm{mCi}$ for radioactive recoils into the gas catcher due to the thickness of the deposit.

\subsection{Helium gas catcher}

The helium gas catcher is a large cylindrical vessel $50 \mathrm{~cm}$ in diameter and $120 \mathrm{~cm}$ in length with one end tapering into a nozzle for extraction of the fissionproduced ions. The large stopping volume of the gas catcher reduces the residual energy of the fission products after they pass through the cover foil and degrader foil (as described below). With their energies greatly reduced, interactions with the helium in the gas catcher lead to final ionization states of $1^{+}$or $2^{+}$for the collected fission fragments. These ions are directed forward with a DC electric field and kept centered in the cell by parallel rings of electrodes with alternating (RF) potential until the gas flow pushes the ions out of the extraction nozzle.

\subsection{Degrader assembly system}

Prior to entering the gas catcher, the ions first pass through a thin gold cover to contain self-sputtering recoil emissions and prevent any flaking of the californium source into the gas catcher. Next, the ions pass through the aluminum degrader foil where much of their residual energy is reduced prior to entering the helium gas catcher.

In the past, a less-than-ideal cylindrical-shaped degrader was utilized due to production limitations. This resulted in non-uniform energy loss as the ions passed through the degrader. With the advent of 3D printing, a new hemispherical degrader was prepared to enable a more uniform energy loss.

\footnotetext{
* Corresponding author: mgott@anl.gov
} 


\section{Degrader assembly preparation}

All of the gas catcher system including the degrader assembly was built and handled to ultra-high vacuum standards as to not affect the purity of the gas; the helium gas is high purity and all contaminants must be kept at the ppb level. The introduction of the new degrader foil presents a possible source of contamination so the use of outgassing materials of any kind is prohibited. The design is such that no epoxies or cements are necessary for the preparation of the degrader assembly. Additionally, the whole setup was assembled and handled in a Class 100 clean bench.

\subsection{Previous degrader assembly}

The previous degrader [3] was cylindrical-shaped and was approximately $10 \mathrm{~cm}$ in diameter and $5 \mathrm{~cm}$ tall. An aluminum base and a thin aluminum ring connected by three thin aluminum posts providing the support structure for the thin aluminum foil. The posts also served to hold and clamp the aluminum degrader foil in place around the circumference of the ring. The thin ring on top held the top degrader foil in place. Figure 1 shows the completed degrader assembly with the aluminum foils installed.

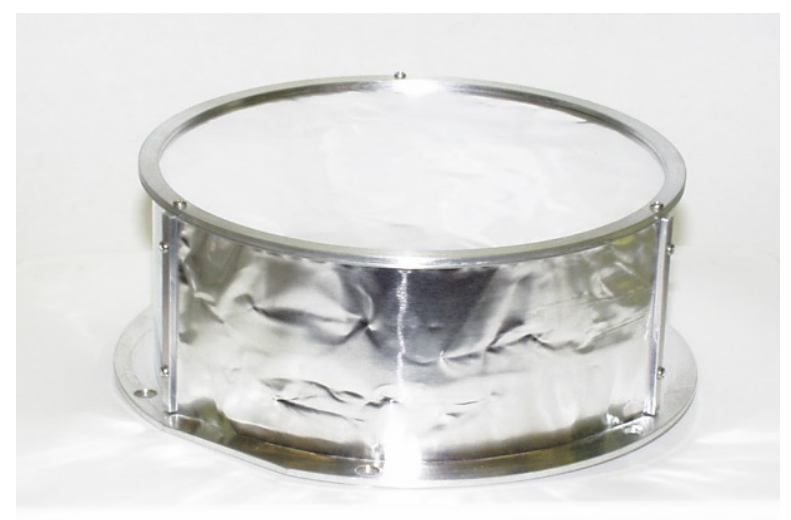

Fig. 1. Previous generation of degrader foil.

\subsection{New degrader design}

The new degrader is a hemispherical design composed of six triangular pieces that screw into a heavy base ring of Inconel steel. Each of the triangular pieces is 3D printed to engineered specifications so that they piece together tightly. The aluminum foil is held in place by a metal gasket that screws into the triangular framing.

The pieces are all cleaned using an acid wash, water rinse, and alcohol rinse. The cleaned pieces are handled with gloves to prevent introduction of contaminants to the surface. All tools are cleaned with alcohol prior to use with this assembly.

\subsection{Assembly of the degrader}

\subsubsection{Preparation of the Aluminum foils}

High purity aluminum foil was purchased from Goodfellow (Coraopolis, Pennsylvania) with an areal density of $1.7 \mathrm{mg} \cdot \mathrm{cm}^{-2}$. The foils were placed between two pieces of weighing paper to prevent tearing as the foils were cut. An in-house fabricated stencil was used to mark the foil to be cut (Figure 2). The foils were carefully cut across the marked lines to create the six foils necessary to fabricate the degrader.

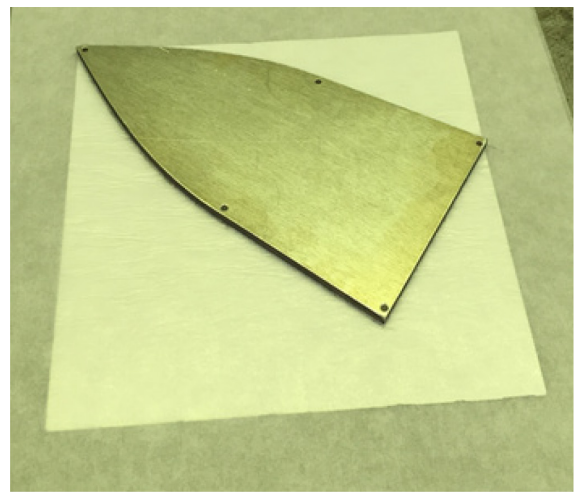

Fig. 2. Stencil placed on top of foil inside of weighing paper.

\subsubsection{Piecing together the sections of the degrader}

The cut foil is placed on top of the 3D-printed frame and, once positioned, the stainless steel gasket is carefully placed on top (Figure 3). A pick is used to punch screw holes in the foil at the base of the frame and then small (sized) screws are used to hold the foil and gasket in place. The gasket is bent around the frame and the screw at the top is attached next following the same procedure. Finally, the two screws on the side are put into place. As these final screws are tightened, the foil stretches slightly and is held tightly in place. The same procedure is followed for all six sections and great care must be taken not to damage/puncture the foil while putting the screws in place.

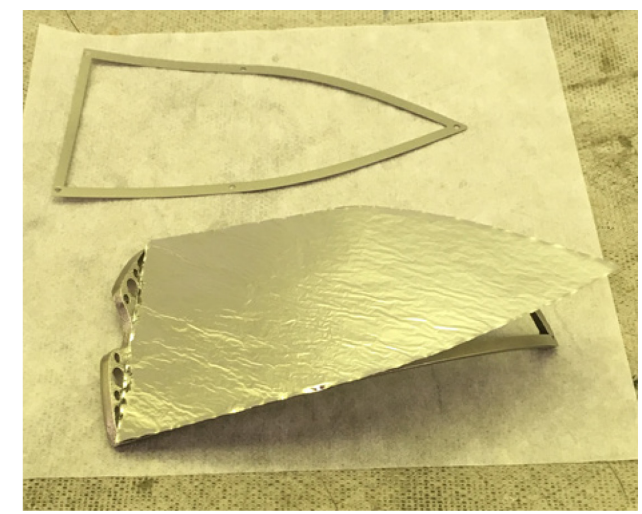

Fig. 3. Placement of foil on frame prior to addition of gasket. 


\subsubsection{Piecing together the degrader assembly}

With the sections completed, the sections were each attached to the base plate using four vented screws. Initially the fragments were screwed in loosely to allow them flexibility to slide in beside one another. Once all sections were installed, a screw was installed at the top to hold them together at the same height and then all the screws at the base were tightened. The final completed degrader is shown in Figure 4.

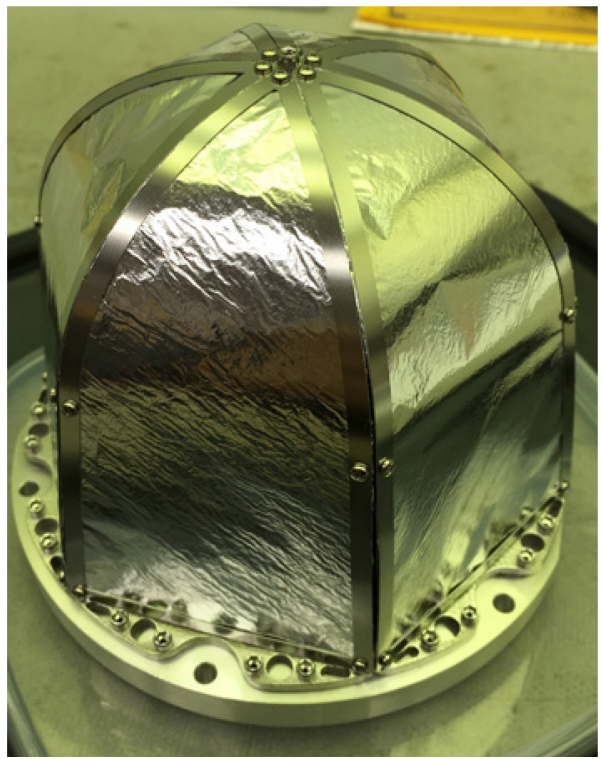

Fig. 4. Completely assembled degrader.

\section{Summary}

The Californium Rare Ion Breeder Upgrade project provides neutron-rich beams for a variety of nuclear physics applications. A new, hemispherical degrader assembly has been prepared to provide a more uniform energy loss for the fission fragments as they enter the helium gas catcher. Care must be taken to avoid damage or contamination during assembly to prevent issues with degrader in the ultra-high vacuum environment in the system.

This material is based upon work supported by the U.S. Department of Energy, Office of Science, Office of Nuclear Physics, under Contract Number DE-AC02-06CH11357. This research used resources of ANL's ATLAS facility, which is a DOE Office of Science User Facility.

\section{References}

1. G. Savard, R.C. Pardo, S. Baker, C.N. Davis et al., Hyperfine Interact. 199, 301 (2011)

2. R.C. Martin, J.B. Knauer, P.A. Balo, Appl. Radiat. Isot. 53, 785 (2000)

3. J.P. Greene, G. Savard, R.C. Pardo, S. Baker et al., Nucl. Instr. Meth. Phys. Res. A 655, 21 (2011) 